\title{
Influence of Design Parameters on the Performance of a Surface Plasmon Resonance Based Fiber Optic Sensor
}

\author{
Yogendra S. Dwivedi • Anuj K. Sharma • B. D. Gupta
}

Published online: 5 June 2008

(C) Springer Science + Business Media, LLC 2008

Erratum to: Plasmonics

DOI 10.1007/s11468-008-9057-z

Unfortunately, the original version of this article has an error in the article title. The first occurrence of the word "Sensor" must be replaced by the word "Resonance" in the article title.

The online version of the original article can be found at http://dx.doi. org/10.1007/s11468-008-9057-z. 